\title{
Clinical Trials in Sepsis - Quo vadis?
}

\author{
Herwig Gerlach
}

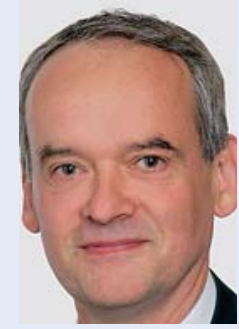

Herwig Gerlach
Korrespondenzadresse Prof. Dr. Herwig Gerlach Klinik für Anästhesie, operative Intensivmedizin und Schmerztherapie Vivantes Klinikum Neukölln Rudower Straße 48 12351 Berlin E-Mail: herwig.gerlach @vivantes.de

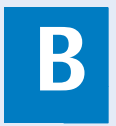

evor man überlegt, wohin ein Weg wohl führen mag, tut man in der Regel gut, sich Gedanken zu machen, wo man steht, und wie die Reise bisher verlief. Wie haben also die letzten 25 Jahre Sepsisforschung in der Intensivmedizin ausgesehen? Sucht man nach großen, randomisierten Studien (randomized clinical trials, RCT), zeigen sich schnell die Grenzen: bis Mitte der 80er-Jahre gibt es im Wesentlichen nur die RCTs zu Steroiden bei septischem Schock, die Eingang in die hochrangige Fachliteratur gefunden haben. Hier wurden also Medikamente geprüft, die es zu diesem Zeitpunkt schon gab, und die keineswegs im Rahmen intensivmedizinischer Forschung entwickelt worden waren. Bei einer der Kernstrategien für die Sepsis, sprich der adäquaten Antibiotikatherapie, sieht es noch düsterer aus: es gab nicht eine einzige hochwertige Studie, die einzelne oder kombinierte Antibiotikatherapien gezielt an septischen Patienten evaluiert hat. Im Gegenteil wurden für entsprechende Studien - etwa bei Pneumonie - Patienten mit schwerer Sepsis teilweise ausgeschlossen.

Erst gegen Ende der 80er-Jahre richteten sich erste Blicke der Pharmaindustrie auf Intensivpatienten mit Sepsis. Grund waren u. a. die bahnbrechenden Entdeckungen zur Pathophysiologie von Infektion, Entzündung und Organversagen. Hier sind als Beispiel Charles Dinarello als Mitentdecker der Interleukine oder Bruce Beutler zu nennen, der das Zytokin Tumor-NekroseFaktor (TNF) beschrieb und hierfür 2011 den Nobelpreis erhielt. Anders als heute waren die initialen RCTs zu Beginn der 90er-Jahre keineswegs zurückhaltend, sondern fast übereilt und teilweise gewagt, wie etwa die Studien zu Anti-Endotoxin oder Anti-TNF, die ja nicht den erhofften Erfolg brachten. Im Nachhinein ist es keine Überraschung, dass solche Initiativen bereits zu dieser Zeit zu mancher Pleite von Unternehmen geführt haben. In den nachfolgenden Jahren wurde man etwas vorsichtiger, Ansätze von adjunktiven Therapien gab und gibt es jedoch auch heute noch reichlich; sie aufzuzählen würde hier den Rahmen sprengen. Praktisch alle diese Studien haben eines gemeinsam: sie zeigten keinen nachhaltigen Vorteil!
Im Jahr 2001 schien dann das Eis gebrochen: die Firma Eli Lilly and Company präsentierte das Produkt Xigris ${ }^{\circledR}$ (Drotrecogin alfa [activated]) mit der PROWESS-Studie, die eine signifikante Sterblichkeitssenkung durch Xigris ${ }^{\circledR}$ von 30,8\% auf 24,7\% bei Patienten mit schwerer Sepsis ergab, die in der Mehrzahl ein Multiorganversagen hatten. Nun gab es zum ersten Mal in der Geschichte der Intensivmedizin ein spezifisches Medikament gegen die schwere Sepsis als gefährlichste Komplikation von Infektionen, die auch heute noch bei erwachsenen Intensivpatienten für die Mehrzahl aller Todesfälle verantwortlich ist. Die Rationale für Xigris ${ }^{\circledR}$ als körpereigenes Protein mit Gerinnungshemmung und konsekutiver Verbesserung von Durchblutung und Organfunktion erschien plausibel, und viele Subanalysen der PROWESS-Studie zeigten eine überzeugende Effektivität. Statt einhelliger Begeisterung kam es jedoch zu teilweise emotionalen und unsachlichen Diskussionen; Gründe hierfür waren neben einer verfehlten Marktstrategie nach Erscheinen der PROWESSDaten die ökonomischen Hindernisse durch den hohen Preis des Medikamentes sowie nicht unerhebliche Eitelkeiten von Intensivmedizinern, die sich plötzlich mit den Konsequenzen evidenzbasierter Medizin auseinandersetzen mussten - also etwas, nach dem man jahrelang gerufen hatte, aber mit dem man eigentlich nicht so recht umgehen konnte, zumal es sich kaum mit der ureigenen „Cowboy-Mentalität“ vereinigen ließ. Diese Widerstände sowie fehlende Wirksamkeit bei anderen Patientengruppen führten dazu, dass von der europäischen Aufsichtsbehörde eine konfirmierende Studie gefordert wurde, in die ausschließlich Patienten mit septischem Schock eingeschlossen wurden (daher „PROWESS-SHOCK“).

Am 25. Oktober 2011 erschien die offizielle Nachricht, dass Xigris ${ }^{\circledR}$ mit sofortiger Wirkung vom Markt genommen wird; Hintergrund war die Entblindung der Daten dieser PROWESS-SHOCK-Studie. Statistisch gab es weder einen Unterschied bei der 28-Tage-Sterblichkeit noch bei dem Risiko einer vermehrten Blutungsneigung. Letztendlich hinterlassen die Daten jedoch mehr Fragen als Antworten: Wieso gab es im letzten 
Jahr der Studie einen unerklärlichen Abwärtstrend der Sterblichkeit auf etwa $20 \%$, und das bei Patienten mit septischem Schock? Wie passt diese niedrige Sterblichkeit zum Umstand, dass - gemessen an der Zahl der Organversagen - die Patienten deutlich kränker waren als in der PROWESS-Studie? Wieso gab es überhaupt keine Blutungsneigung durch das Medikament? Muss daher an jeglicher Wirksamkeit gezweifelt werden, wenn man berücksichtigt, dass für diese Studie eine neue Charge von Xigris ${ }^{\circledR}$ benutzt wurde? Wie passt dies zu den Ergebnissen zweier großer multinationaler Register mit insgesamt fünfstelliger Patientenzahl, bei denen sich der sterblichkeitssenkende Effekt von Xigris ${ }^{\circledR}$ in hochsignifikanter Weise bestätigt hat? Ist es müßig, überhaupt noch zu spekulieren? Die Empfehlungen in Sepsis-Leitlinien für Xigris ${ }^{\circledR}$ sind inzwischen ersatzlos gestrichen! Ist das „Aus“ für Xigris ${ }^{\circledR}$ auch das Ende industriell geförderter Sepsisforschung, weil Intensivpatienten offensichtlich zu „riskant“ sind? Kurzum: waren die letzten 10 Jahre vertane Zeit?

Zumindest die letzte Frage muss man ausdrücklich verneinen! Auch wenn die „Xigris ${ }^{\circledR}$-Story“ ernüchternd ist, sie ist nicht das Surrogat jeglicher Sepsisforschung. Es gab viele andere Ansätze, und der „hype“ nach der PROWESS-Studie war richtig und wichtig. Die Gründungen der Surviving-Sepsis-Campaign (die es ohne PROWESS so vermutlich nicht gegeben hätte) sowie zahlreicher anderer Gesellschaften zum Thema Sepsis sind nur ein Beispiel, und gerade in Deutschland sprechen die Erfolge etwa des Kompetenznetzwerkes Sepsis (Sepnet) für sich. Insofern stehen wir nicht am Anfang des Weges, sondern mitten am Hang des Berges. Zugegeben, etwas wackelig zurzeit, aber ein Grund mehr, nun den Blick nach vorne zu richten und nachzudenken, wo man die Pflöcke für den Weg nach oben einschlagen soll. Die Lehren der Vergangenheit sind hierfür wichtig, und so könnte man - ohne jeglichen Anspruch auf Vollständigkeit - die strategische Ausrichtung für die Zukunft etwa durch die folgenden Ziele definieren:

- Sepsisforschung ist dringend und wichtig! Nicht primär für die Intensivmediziner, sondern für die Patienten, wenn man die immer noch bedrohlichen demografischen Daten betrachtet.

- Dies muss durch mehr Öffentlichkeitsarbeit, Einbindung der Kostenträger und stärkere Bemühungen um öffentliche Förderungen unterstützt werden.

- Eine aktive Beteiligung der Industrie ist auch für die Zukunft unabdingbar! Zu glauben, die Aufgaben bei den technischen, finanziellen und juristischen Ansprüchen für die Entwicklung innovativer Produkte ohne Industrie bewältigen zu können, ist naiv.
- Nur neue adjunktive Verfahren zu prüfen, ist gefährlich! Gerade für die „klassischen“ Bereiche wie Antibiotika sind neue Ansätze längst überfällig; hier ist die Entwicklung in den letzten Jahren dramatisch abwärts gerichtet. Wichtig dabei ist, dass diese Studien spezifisch für Patienten mit schwerer Sepsis geplant werden und die Intensivmedizin nicht, wie in der Vergangenheit, als „Nebenspielplatz“ betrachtet wird.

- Die notwendige Selektion der Patienten bietet zahlreiche Fallstricke: es gibt nicht den typischen Sepsispatienten! Wir haben es mit Unterschieden zu tun, was Geschlecht, ethnische Herkunft, Strukturen, Kulturen und Krankheitsbilder angeht, und das muss bei der Planung zukünftiger Studien viel mehr und von vornherein berücksichtigt werden.

- Prospektive RCTs sind wichtig, aber bergen stets die Gefahr zu starker Selektion und „Exklusivität“. Bei der jüngst abgeschlossenen MAXSEP-Studie des Sepnet wurden nur 11,4\% der gescreenten Patienten eingeschlossen (und das waren sicherlich nicht alle septischen Patienten!), und bei der noch laufenden HYPRESS-Studie sind es nur 3,8\%.

- Parallele Observationsstudien und Register sind unverzichtbar, da sie repräsentativer sind. Vorsicht ist allerdings geboten, hierbei mit statistischen „Tricks“ Beweise zu kreieren, wo es keine gibt. „Metaregressionen“ oder „Quasi-Randomisierungen“ können gefährlich werden, wenn sie für gepoolte Daten benutzt werden; sie sind und bleiben bestenfalls deskriptiv.

- Wir Kliniker müssen uns selbst den Rahmen schaffen, um solche großen Datengrundlagen zu generieren. In Kanada und Australien wurde gezeigt, dass es möglich ist, solche Organisationen zu bilden, und das deutsche Sepnet ist auf einem vergleichsweise guten Weg.

Alles in allem gibt es keinen Grund, den Kopf hängen zu lassen, was die zukünftige klinische Sepsisforschung in Deutschland betrifft. Für die Industrie sind die Zeiten sicherlich im Moment hart, und dementsprechend ist das Engagement zurückhaltend. Ein Grund mehr für uns Kliniker, Aktivität zu zeigen, sonst geraten wir in Gefahr, den Beweis, klinische Forschung mit einem hohen Maß an Autarkie und Kreativität betreiben zu können, schuldig zu bleiben. 